\title{
Improving Choice Model Parameter Estimates by Jointly Modelling the SP Choices with Corresponding Elicited Certainty Ratings
}

Khandker Nurul Habib, Ph.D., P.Eng

Associate Professor, Department of Civil Engineering

University of Toronto

Email: khandker@civ.utoronto.ca

\section{Citation:}

Habib, K.M.N. 2017. Improving choice model parameter estimates by jointly modelling the SP choices with corresponding elicited certainty ratings. Transportation Research Part A (Forthcoming) 


\begin{abstract}
The paper proposes a closed-form econometric model of joint SP choices and corresponding elicited certainty ratings. The connection between the SP choices and corresponding elicited certainty ratings is modelled through an entropy-based measure of SP choice task complexity. Empirical applications of the proposed model are presented by using two SP survey datasets collected in Vancouver and Toronto. Empirical models reveal that the SP choice task complexity, measured through SP choice entropy, directly influences SP certainty ratings. Such direct relationship proves to be important through establishing an endogenous relationship between them. It is clear that capturing such endogeneity improves the efficiency of parameter estimates of the SP choice model. However, the level and extent of such benefit gains vary by the nature and complexity of the SP survey. Empirical investigation presented in the paper proves that both efficiency gain and higher goodness-of-fit are probable with the choice contexts with higher number of choice alternatives. However, even for the smaller number of choice alternative case, efficiency in parameter estimates can be increased by proposed joint model formulation.
\end{abstract}

Key Words: Stated preference survey, elicited confidence rating, choice model, mode choice 


\section{Introduction}

Stated Preference (SP) survey or SP experiment is now an unavoidable tool for investigating consumer behaviour in many circumstances (Carson and Louviere 2011). Such investigations include uncovering apparently obscure marginal rate of substitutions of different product attributes and understanding demand of new product/choice alternatives. Application of the SP survey in transportation planning and travel demand modelling is now widespread and widely accepted. Our increasing reliance on SP to investigate consumer choice behaviour demands realistic depictions of choice scenarios in SP experiments. To some extent, the validity of any SP survey depends on how consistent the stated choices are compared to the actual choices of similar types in similar real contexts.

Since SP surveys deal with hypothetical scenarios, there is always a dilemma in using SP data because of the inherent uncertainty of hypothetical choices. From a respondent's point of view, such uncertainty stems from the apparent hypothetical nature of the experiment, where the respondent will not experience the immediate outcome of the choices. So, the choice modellers need to worry about the extent and nature of such uncertainty perceptions of the respondents that eventually affect the quality of data. A direct way of measuring such uncertainty is collecting respondent-elicited certainty ratings of their SP choices. Respondents can elicit their certainty through a Likert scale after each SP choice task. Efforts to improve the SP choice model parameter estimates through the exploitation of elicited certainty ratings are evident in the literature. However, concrete evidence of the nature and extent of such improvement on choice model parameter estimates is not yet fully clear yet.

To contribute to this topic, this paper proposes a joint econometric model for SP choice and corresponding elicited certainty ratings. Considering that the elicited certainty ratings are observed outcomes of a latent perception of SP choice task complexity, it uses SP choice entropy as a measure to establish the link between the SP choices and corresponding perception of certainty of the choice makers. Allowing unrestricted correlations between the SP choices and the corresponding elicited certainty rating, the proposed model allows investigating the ways to improve the SP choice model parameter estimates. Two empirical applications of the model are presented by using two datasets collected in Toronto and Vancouver.

The paper is organized as follows: Section 2 presents a brief literature review on the role and use of elicited certainty rating. Section 3 presents the econometric modelling frameworks. Section 4 presents a brief description of the datasets used for empirical investigations. Section 5 presents the discussions on empirical models. Finally, the paper concludes with a summary of key findings and recommendations for further research.

\section{Literature Review}

A vast amount studies on various aspects of SP survey are documented in the literature. Rose and Bliemer (2009); Louviere et al. (2010); Rose and Bliemer (2013); Hensher and Ho (2014) are some recent examples that present comprehensive reviews of various aspects of SP surveys including scenario design methods, sample size determination, non-attendance etc. However, the research on the potential use of users' perceived certainty (of SP choices) information is 
relatively new even though the preference uncertainty in SP surveys has been a recognized area of interest for a long time. Preference uncertainty in SP surveys has always been a concern in non-market valuations or welfare analysis in environmental economics (Shaikh et al. 2007). So, most of the approaches of uncertainty elicitation and usage of certainty rating information to reduce biases in SP survey are primarily for binary discrete choice experiments or contingent valuation studies. In many cases, these are difficult to generalize for multi-alternative SP contexts (Dekker et al. 2016). However, recent advances in multi-attribute and multi-alternative SP experiments reignited interests in elicitation of preference uncertainty in SP experiments and the use of such elicited information in improving SP choice models.

Elicitation process of SP choice (un)certainty has long been debated by the researchers. Manski $(1995,1999)$ suggested direct questions for the respondents to elicit (as a percentage or likelihood of) their probabilistic assessment of hypothetical preferences. Bemmaor (1995) argues that uncertainty elicitation should not be so strict in terms of continuous percentage or likelihood-based assessment, and suggested that it should be qualitative in nature, e.g., "very certain," "probably not," "certainly not," etc. Li and Mattsson (1995) used the continuous certainty measure (as percentage $0 \%$ to $100 \%$ ) for the elicitation of contingent valuation choices. They assumed that the respondents know their true preferences and hence can be very certain in eliciting certainty in percentages. They used a threshold for the elicited certainty to further strengthen/correct the yes/no responses to the hypothetical choice tasks and thereby adjusted their choice model parameter estimates. A criticism of continuous scale is that the assumption of accurate knowledge of preferences of respondents is a very strong assumption. Also, using elicited responses to further modify SP choices is very arbitrary. Reddy et al. (1995), Loomies and Ekstrand (1998) and many others used qualitative measures for certainty-rating elicitation of their willingness-to-pay study. Hanemann et al. (1999) recommended the procedures of collecting respondent's perception of uncertainty in the form of interval scale than in the form of strict numbers.

In general, elicited certainty ratings of SP experiments are procedural invariants that capture choice task complexity of SP choice tasks (Brouwer et al. 2010). However, preference uncertainty contributes to a big portion of hypothetical biases in SP choice experiments (Champ and Bishop 2001). In practice, both qualitative and quantitative ratings of SP choice certainty are used and often such information are used directly or indirectly in the investigation of SP choice data. One common way of incorporating such elicited certainty ratings into the SP choice models is applying a threshold of certainty to screen out similar response data to the model. Such practice of considering threshold to screen out similar response data is to eliminate SP choices that have very low certainty (Champ et al. 1997; Ethier et al. 2000; Champ and Bishop 2001; Lundhede et al. 2009).

However, Norheim (2001) argues that scrapping of low- certainty SP choices in order to remove outliers from the SP data are counterproductive as low- certainty responses are not necessarily wrong choices. In fact, low- certainty choices reflect the respondent's ambivalence to the choice tasks because of the task complexity, and Arentze et al. (2003) found that choice task complexity may not always affect the quality of SP survey data. Obviously, the definition of 'data quality' is a subjective issue and difficult to generalize. However, Caussade et al. (2005) and Olsen et al. (2011) found that choice complexities, resulting from combinations of SP choice attributes and 
their levels, affect choice variance or consistency. Shaikh et al. (2007) report that accommodation of uncertainty in the choice model may result in better goodness-of-fit of the choice model, but also introduce additional variance. So intuitively, it would be better using the elicited certainty ratings into the SP choice model estimation process to reflect the choice uncertainty into the choice model parameter estimates. Lundhede et al. (2009) investigated various ways of accommodating respondents' elicited SP certainty ratings. They found that specifying choice model scale parameters as a function of elicited choice uncertainty (certainty rating) may not improve the performance of the choice model, but provides a structurally stable way of accommodating such additional information in the SP investigation.

Hensher et al. (2012) particularly investigated factors that may influence the elicited SP choice certainty ratings by using the ordered probability model. They found that the elicited certainty rating on any SP choice task is influenced by choice task complexity, which may be attributed to the number of alternatives, the number of attributes and their levels in the choice tasks. They recommend that the perception captured through the elicited certainty rating can be used to improve choice tasks. However, they did not investigate how such information can be exploited to enhance the SP choice model.

Beck et al. (2013) investigated how the SP choice certainty rating can be used to reduce biases in SP choices. They used a multinomial logit model with parameterized scale function. They found that the elicited certainty indices of SP surveys can be used to explain the scale parameter of the SP choice model. They identified that the lack of a proper theoretical background of accommodating elicited certainty ratings into choice modelling is the main reason of difficulty in explaining the influences of such ratings on SP choices. They found that specifying the SP choice model scale parameter through the elicited certainty ratings might result in different parameter estimates based on different functional forms. So, their empirical investigation did not reveal any definitive conclusions on the use of elicited confidence rating information in the SP choice model estimation process.

Rose et al (2015) investigated the endogeneity between elicited response certainty and SP choice through applications of discrete choice models. Their empirical model captured such endogeneity by estimating separate choice models for different levels of elicited certainty. Splitting the dataset based on certainty levels can become arbitrary as the modeller needs to a threshold of the certainty level. In this application, they used certainty level 6 (in a 10-point scale) as the threshold to split the dataset for estimating two sets of choice models. Even if the threshold level is selected arbitrarily, the loss of data (the responses with lower certainty level than the threshold) is evident as their empirical investigation reveal that marginal sensitivities of choice model attributes are indifferent to the elicited certainty level.

Dekker et al. (2016) proposed an integrated latent variable choice model for exploiting elicited certainty ratings into the discrete choice model estimation process. Using the latent variable modelling approach, they used personal attributes of the respondents to explain the latent certainty ratings measured through the elicited confidence ratings as ordered regression model. The latent variable model is jointly estimated with the SP choice model, where the latent variable is also used to parameterize the scale and alternative-specific-constants ('asc') of the SP choice model. The resulting mixed discrete choice model is then estimated by using Bayesian estimation 
technique. In order to further capture the intuitive relationships between perceived certainty of the respondents and their SP choice, they investigated the use of SP choice entropy as one of the explanatory variables of the latent certainty rating model. However, the tautological relationship between SP choice entropy and the scale parameter posed choice model estimation challenge. So, it did not prove the improved model fit (likelihood value) or statistical significance of the parameter estimates.

This paper proposed a definable and intuitive approach to addressing the link between SP choices and corresponding elicited certainty ratings with the facilitation of a closed form choice model formulation. Considering that the certainty elicitations follow the SP choice tasks, it assumes that the task complexity of the SP choice directly influences the corresponding elicited certainty ratings. However, it also assumes there could be correlated unobserved factors that influence both SP choices and the corresponding elicited certainty ratings. The next section explains the econometric structure of the proposed modelling framework.

\section{Modelling Framework}

For the purpose of explaining the methodology proposed in the paper, let us assume that the SP choice experiment is composed of a discrete choice experiment followed by the SP certainty rating. Each respondent (suppressing the respondent's identifier in the equation) faces ' $S$ ' number of SP scenarios of ' $J$ ' alternative discrete choices and each scenario receive a certainty rating on a scale between 1 (totally uncertain) and $K$ (most certain). Under the Random Utility Maximization (RUM) theory, the total utility $\left(U_{j}\right)$ of any discrete choice alternative $j$ is:

$U_{j}=\sum(\beta x)_{j}+\varepsilon_{j} \quad j=1,2, \ldots . . J$

here, the subscript $j$ indicates alternative $j$

In an SP experiment, it is obvious that the choice of alternatives and corresponding certainty ranking is intricately related. Let us assume that the random components of the choice model utility follow Type I Extreme Value distribution with scale parameters $\mu_{c}$. This results in a multinomial logit model of:

$$
P(j)=\exp \left(\mu_{c} V_{j}\right) / \sum_{m=1}^{J} \exp \left(\mu_{c} V_{m}\right)
$$

here, the subscript $j$ refers to any specific discrete choice alternative.

Alternatively, for a typical example of $J$ alternatives with multiple nests, the choice probability of any alternative in nest 1 becomes:

$$
\begin{aligned}
& P(j)=\exp \left(\mu_{c_{-} n} V_{j}\right) / \sum_{n_{-} m=1}^{n} \exp \left(\mu_{c_{-} n} V_{n m}\right) \\
& \times \frac{1}{\mu_{c_{-} n}} \ln \left(\sum_{n_{-} m=1}^{n} \exp \left(\mu_{c_{-} n} V_{n_{-} m}\right)\right) / \sum_{n=1}^{N} \frac{1}{\mu_{c_{-} n}} \ln \left(\sum_{n_{-} m=1}^{n}=\exp \left(\mu_{c_{-} n} V_{n_{-} m}\right)\right)
\end{aligned}
$$


here, subscript $n$ indicates any specific nest considering that the total number nests in $N$; subscript $m$ indicates any specific alternative in nest $n$ considering that the total number of alternatives in any nest is $M$.

Similarly, let us assume that the stated certainty rating has an underlying continuous measurement $\left(R_{k j}\right)$ that is elicited along an ordered scale of certainty rating:

$R_{k j}=C_{k j}+\xi_{k j}$

here, subscript $k j$ refers to the elicited certainty rating corresponding to the stated choice of alternative $j$

$C_{k j}$ indicates a linear-in-parameter function of variables and their coefficients

$\xi_{k j}$ is the standard normal random component.

Considering that $R_{k j}$ is a continuous latent variable, the elicited certainty rating takes the value of $\left(I_{r}\right)$ if $R_{k j}$ falls within the threshold limits of $I_{r}$. Considering that the certainty rating is elicited along an axis from $r$ equal to 1 to a positive value $R$, the corresponding ordered probability model takes the form of:

$P\left(I_{r}=r \mid C_{k j}\right)=\Phi\left(\mu_{r}-C_{k j}\right)-\Phi\left(\mu_{r}-C_{k j}\right)$

here, $\Phi($. . ) indicates the cumulative density function of standard normal distribution.

It is clear that the choice model probabilities (equation 2 and 3), as well as the certainty rating probability (equation 5), represent marginal probabilities. Any marginal probability can be converted into an equivalent standard normal probability. Hence, inverting any such marginal probability into an equivalent standard normal variable will result (Lee 1983):

$\eta_{j}=\Phi^{-1}(P(j))$

here, $\eta_{j}$ refers to a transformed standard normal random variable corresponding to the stated choice of any alternative $j$.

Now, if the choice certainty rating follows the stated choice making, it is understandable that there are obvious endogenous relationships between the stated choice of any alternative and corresponding certainty rating. Such endogenous relationships can be expressed in two ways:

1. the random components of stated choice utility and elicited certainty rating are correlated.

2. there is a relationship between the SP choice uncertainty and the choice certainty rating. This paper tackles both of these ways simultaneously. Considering the first relationship, the obvious way is to consider $\eta_{\mathrm{j}}$ and $\xi_{k j}$ are bivariate standard normal distributed with correlation $\left(\rho_{j}\right)$. Such assumption results in the joint probability of the stated choice and elicited certainty rating as:

$P(j \& j k)=P(j) \times\left[\begin{array}{c}\Phi_{2}\left(\eta_{j},\left(\mu_{r}-C_{k j}\right), \rho_{j}\right) \\ -\Phi_{2}\left(\eta_{j},\left(\mu_{r-1}-C_{k j}\right), \rho_{j}\right)\end{array}\right]$

For any individual $i$, considering $J$ number of SP choice alternatives for one scenario $s$ and corresponding $R$ levels of certainty rating, the likelihood function $\left(\mathrm{L}_{\mathrm{isj}}\right)$ become: 


$$
L_{i s j}=P(j)_{i} \times\left[\begin{array}{c}
\left(\Phi_{2}\left(\eta_{j},\left(\mu_{1}-C_{k j}\right), \rho_{j}\right)\right)^{\delta_{0}}+\ldots \ldots \ldots \ldots \\
\quad+\left(\Phi_{2}\left(\eta_{j},\left(\mu_{r}-C_{k j}\right), \rho_{j}\right)-\Phi_{2}\left(\eta_{j},\left(\mu_{r-1}-C_{k j}\right), \rho_{j}\right)\right)^{\delta_{r}}+\ldots . \\
+\left(\Phi_{2}\left(\eta_{j},-\left(\mu_{R}-C_{k j}\right),-\rho_{j}\right)\right)^{\delta_{R}}
\end{array}\right]_{i}
$$

here, the subscript $i$ refers to any individual, $s$ indicates a specific scenario and $j$ indicates a chosen alternative discrete SP choice.

$\delta$ is an indicator; $\delta_{r}=1$ if certainty rating is $\mathrm{r}$ and o otherwise and $\mathrm{r}$ varies from 1 to $\mathrm{R}$.

$\Phi_{2}$ (..) indicates the cumulative density function of bivariate standard normal distribution.

Now considering $\mathrm{J}$ is the number of alternatives, the generalized likelihood function becomes:

$$
L_{i s}=\prod_{j=1}^{J}\left(L_{i s j}\right)^{\delta_{j}}
$$

here, $\delta_{j}$ is an indicator; $\delta_{j}=1$, if alternative $j$ is chosen and 0 otherwise.

This presents a discrete-ordered econometric structure. Finally, for each respondent facing $\mathrm{S}$ number of SP scenarios and considering a total of $P$ number of respondents, the sample likelihood (L) becomes:

$$
L=\prod_{i=1}^{P}\left(\prod_{s=1}^{S} L_{i s}\right)
$$

This econometric formulation represents a panel data joint discrete-ordered choice model, where the discrete choice explains the SP choices, the ordered choice explains the SP certainty ratings and the panel version represents the fact that each respondent has multiple SP choice and certainty rating observations. Under such joint econometric formulations, this paper investigates the hypothesis on the joint SP choice and certainty rating model. The SP certainty rating is stated after the SP choices are made, and one can easily argue that uncertainty derived from the choice complexity of an SP scenario directly influences the corresponding certainty rating. Choice complexity of a discrete choice can be measured by the measure of entropy ${ }^{1}$ as follows (Shannon 1948; Swait and Adamowicz 2001a and 2001b; Dekker et al. 2016; Habib et al. 2014):

$$
H_{s}=\sum_{j=1}^{J}-P_{j} \ln \left(P_{j}\right) \text { for } P_{j}>0
$$

here, $H_{s}$ refers to Shannon's entropy measure of an SP choice task

\footnotetext{
${ }^{1}$ As one the reviewers identify that entropy is not exactly complexity; it is just a measure of how close choice probabilities are within a choice task. There are other things that could make a choice task "complex" that are not necessarily a function of how close the choice probabilities are. For example, the respondent could find the task very easy, but still have two alternatives that are very close. This is worth noting.
} 
$P_{j}$ refers to modelled choice probability as explained in equations (2) and (3).

$H_{s}$ is a positive term that can vary between 0 (perfectly certain choice) and the logarithm of the inverse of the number of feasible choice alternatives (perfectly random choice). We consider the simplest function of $C_{j k}$ would be a linear or quadratic function as follows (Swait and Adamowicz 2001a and 2001b):

$C_{k j}=\theta H_{s}+\theta^{\prime} H_{s}^{2}$

here, $\theta$ and $\theta^{\prime}$ are coefficients.

A quadratic function is assumed in this investigation; however, logarithmic or higher-order polynomial functions are also possible. In any case, the joint model has a closed form and can be estimated by using the classical maximum likelihood estimation routine. In this paper, the models are estimated by a program written in software GAUSS using its standard maximum likelihood estimation routine, Maxlike 5.0 (Aptech 2015). However, alternative choice model formulations, e.g., mixed logit model would be very straightforward to accommodate, but would require either simulation-assisted estimation or the Bayesian estimation technique.

\section{Datasets for Empirical Investigation}

Two datasets collected in two Canadian cities for two different choice processes are used for the empirical investigation of this paper. One dataset is from an SP survey of mode switching behaviour under alternative transit service attributes conducted in the City of Toronto in 2012 (defined as "Toronto SP survey" hereafter). The other datasets are from an SP survey of investigating commuting mode choice behaviour in the Greater Vancouver Region conducted in 2012 (defined as "Vancouver SP survey" hereafter).

Both surveys were conducted by marketing research companies who used the random sampling technique, and the combined marketing research panel and active telephone recruitment process. While the "Toronto SP survey" is well-documented in literature (Idris et al. 2014), the "Vancouver SP survey" was part of a policy investigation for a transit agency and so was not published anywhere yet. Figure 1 presents snapshots of the SP schemes of both surveys. Both these surveys used D-efficient SP design. Each SP scenario of the Toronto survey has three discrete choice options to choose from. These are:

1. Not switching currently commuting mode (driving a private car).

2. Switching to transit.

3. Switching to other modes, e.g., non-motorized modes.

Each respondent faced all three options in all six scenarios of the SP experiment. Each SP scenario of the Vancouver survey has a maximum of five choice alternatives (depending on the feasibility of alternative transit options). These are:

1. Regional transit (West Coast Express) with Park \& Ride access.

2. Regional transit (West Coast Express) with walk access.

3. Other transit (Evergreen transit) with Park \& Ride access.

4. Other transit (Evergreen transit) with walk access.

5. Private car.

Toronto survey deals with variations in transit service attributes (travel time, waiting time, schedule delays, information provision, etc.) to capture response in terms of switching to transit 
or staying status quo to switching to other modes than transit. The Vancouver survey presents full variations of all levels of service attributes of all five modes to the respondents to choose among multiple-choice alternatives. Service attributes of transition options include travel time, fare, access time, waiting time, peak and off-peak service frequency, etc. However, the common thing between both surveys is that certainty rating questions are asked after each SP scenario in both surveys. So, in both surveys, each respondent made 6 SP choices and elicited 6 certainty ratings.

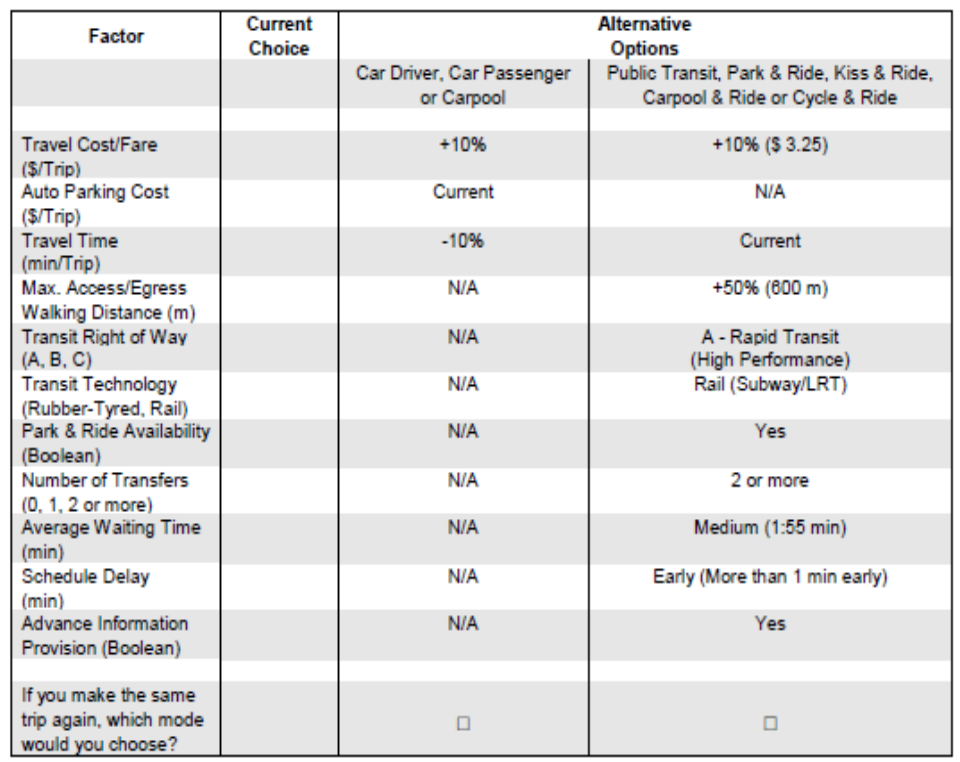

Certainty rating (willingness to comply in reality):

\begin{tabular}{|c|c|c|c|c}
\hline $\begin{array}{c}\text { Very } \\
\text { Weak }\end{array}$ & $\begin{array}{c}\text { Moderately } \\
\text { Weak }\end{array}$ & Neutral & Moderately & Very \\
Strong & Strong \\
$\square$ & $\square$ & $\square$ & $\square$ & $\square$ \\
\hline
\end{tabular}

\begin{tabular}{|c|c|c|c|c|}
\hline & $\begin{array}{l}\text { West Coast Express } \\
\text { Park \& Ride Access }\end{array}$ & $\begin{array}{l}\text { West Coast Express } \\
\text { Transit/Walk Access }\end{array}$ & & Auto \\
\hline Peak Service & \multicolumn{2}{|c|}{$\begin{array}{l}5 \text { morning trains every half hour } \\
\text { (departing Mission City at 5:25-7:25AM) } \\
5 \text { evening trains every half hour } \\
\text { (departing Waterfront at 3:50-6:20 PM) }\end{array}$} & $\begin{array}{l}* * \text { remove these collumns if Evergreen/Skytrain } \\
\text { local transit is infeasible }\end{array}$ & - \\
\hline Off-Peak Service & \multicolumn{2}{|c|}{$\begin{array}{l}\text { Scheduled bus service ( } 2 \text { midday buses in each } \\
\text { direction, } 3 \text { eastbound evening buses) } \\
\text { (102 minute travel time) }\end{array}$} & & - \\
\hline Able to find a seat & \multicolumn{2}{|c|}{ Every day } & & - \\
\hline $\begin{array}{l}\text { Late to work by } 10 \\
\text { minutes or more }\end{array}$ & \multicolumn{2}{|c|}{ Once every six months } & & Once a month \\
\hline $\begin{array}{l}\text { Likelihood of } \\
\text { finding parking }\end{array}$ & High & - & & High \\
\hline Travel Time (Peak) & $\begin{array}{l}100 \text { min } \\
(15 \text { drive } \\
+75 \text { train } \\
+10 \text { walk })\end{array}$ & $\begin{array}{l}115 \text { min } \\
(30 \text { transit } \\
+75 \text { train } \\
+10 \text { walk })\end{array}$ & & $43 \mathrm{~min}$ \\
\hline Trip Cost & $\begin{array}{l}\$ 11.25 \text { fare }+\$ 3 \text { park } \\
\quad+\text { gas } \$ 1.36 / \text { litre }\end{array}$ & $\$ 11.25$ fare & & $\begin{aligned} & \$ 13 \text { park } \\
+ & \text { gas } \$ 1.36 / \text { litre }\end{aligned}$ \\
\hline $\begin{array}{l}\text { Your current mode } \\
\text { choice: }\end{array}$ & 0 & 0 & 0 & 0 \\
\hline
\end{tabular}

Certainty rating (willingness to comply in reality): $\begin{array}{llll}\text { (unsure) } 1 & 2 & 3 & 4 \text { (certain) }\end{array}$

\section{Vancouver Survey}

\section{Figure 1: Snapshots of SP survey parts of two surveys}

The Toronto survey datasets that were available for this study were composed of 862 completed responses. Each of the 862 respondents was private car users, and responded to a total of six SP scenarios through choosing "not changing mode (continue using the private car)," "switching to 
transit" and "switching to other modes, e.g., non-motorized modes." The Vancouver dataset includes a total of 586 completed responses. Each of the 586 respondents faced six SP scenarios and provided a certainty rating for each SP scenario.

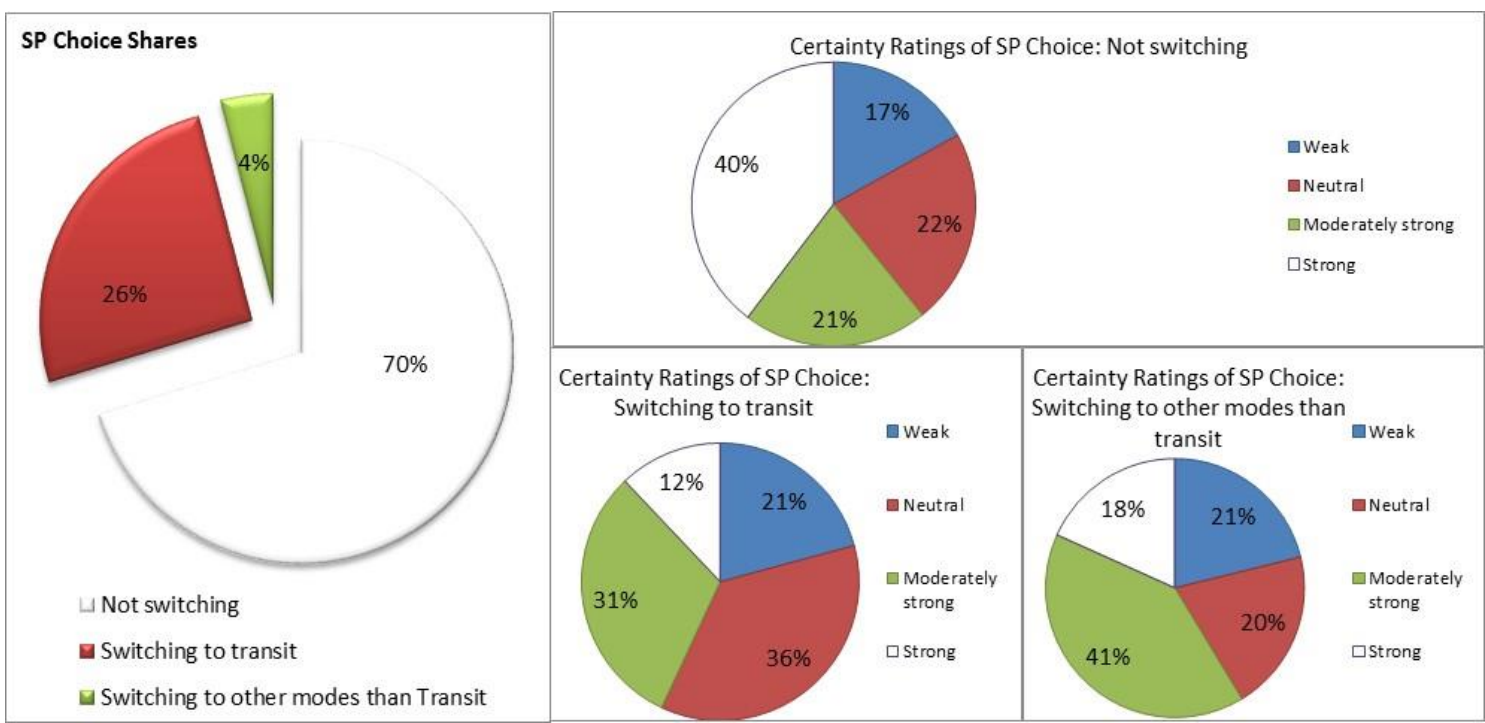

\section{Toronto Survey}

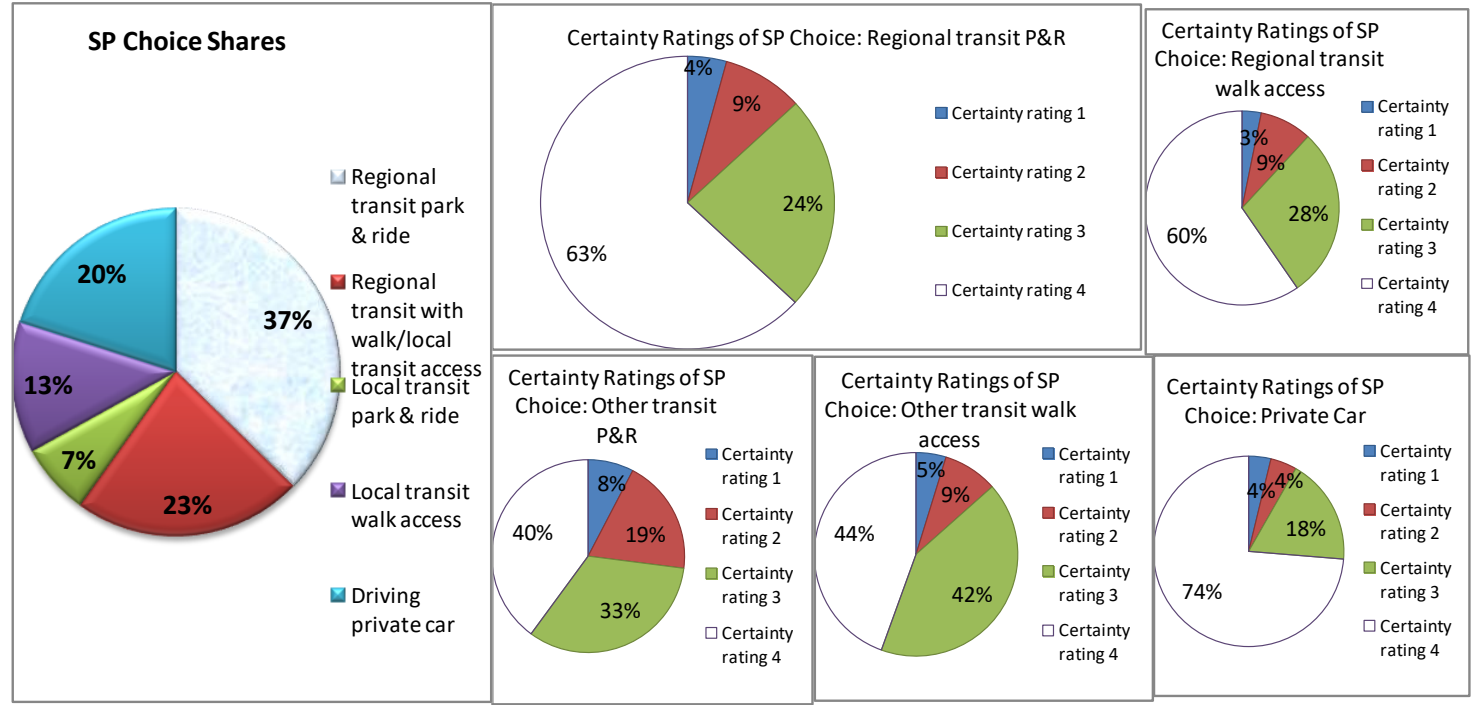

\section{Vancouver Survey}

\section{Figure 2: Summaries of Choice Proportions and Certainty Ratings}

Two available survey datasets for this study have slightly different objectives along with slightly different choice contexts, However, in both surveys, respondents were asked to rate their confidences on the SP choice they made. SP confidence (certainty) rating options followed each SP choice task. So, each respondent in both surveys faced 6 SP scenarios and 6 corresponding certainty rating elicitation. However, two surveys used a different style of certainty rating elicitation. Toronto survey uses the 5 point ratings as: 'very weak', 'moderately weak', 'neutral', 
'moderately strong' and 'very strong'. However, the Vancouver survey uses a 4 point numerical rating starting from 1 as the very weak or 'unsure' and 4 as the strong or 'certain'. In case of Toronto survey, the number of observations with 'moderately weak' ratings are found very low (only 1 complete and couple of incomplete records). So those records are removed and the resulting dataset has the elicited certainty ratings with 4 options: 'weak', 'neutral', 'moderately strong' and 'very strong'.

SP choice data along with certainty rating information of both surveys provide unique datasets for the current investigation. From survey methodological points of view, both surveys used the same design technique (D-Efficient). However, in terms of the choice contexts of two surveys, the Toronto survey is simpler than the Vancouver survey. Figure 2 presents summaries of choice proportions and distribution certainty ratings of two surveys. The Toronto survey has a smaller number of choices than the Vancouver survey. Also, the Toronto survey presented the Revealed Preference (RP) mode attributes besides the SP scenarios that make the SP choice making very straightforward and pivoted to the current choice. However, the Vancouver survey presents the full SP profile without showing the RP mode attributes besides the SP scenarios.

Both surveys have dominant choices. The dominant choice (not switching) is higher in Toronto survey than that of the Vancouver survey (regional transit P\&R). In terms of certainty ratings, both surveys show highest levels of certainty of the dominant choices. However, unlike the Toronto dataset, all non-dominated choices also have the highest certainty ratings in Vancouver dataset. In the case of Toronto dataset, the respondents do not have the highest certainty ratings on non-dominated choices. Overall, both datasets provide different distributions of choice and certainty ratings. The following section investigates the hypothesis regarding the role of certainty rating in improving choice models developed by using SP survey data.

Having two slightly different datasets for this investigation allows us testing the proposed methodology of enhancing SP choice model parameter estimation for two slightly different contexts. While having exactly same survey data from two study area could also be an interesting opportunity of testing robustness of the proposed methodology, I believe, having slightly different dataset, in fact, expands the application contexts instead. Nevertheless, it will be beneficial to apply the same methodology in different other SP datasets too.

\section{Empirical Model}

Joint discrete-ordered model of SP choices and certainty rating are estimated for both datasets. For the Toronto dataset, discrete mode-switching choices are modelled as a multinomial logit model. In the case of the Vancouver dataset the discrete mode choice model is a nested logit model considering private car, regional transit with park \& ride access, and local transit park \& ride access modes nested in a group as opposed to independent modes of local transit walk access, regional transit with walk access and regional transit with local transit access. Figure 3 presents the choice model alternatives of two datasets. A number of alternative specifications for the discrete and ordered model components are tested considering sign expectations and statistical significance. A 95\% confidence limit is considered for evaluating the statistical significance test. In all cases, it becomes clear that the choice model entropy functions have direct as well as indirect influences on elicited certainty ratings. Direct influence is captured 
through the parameters of linear as well as quadratic entropy function elements in the ordered probit covariate function. Indirect influence is captured through the correlations between unobserved factors affecting the SP choices and corresponding uncertainty rating elicitations. Goodness-of-fit of the models are measured by calculating rho-squared values of choice model components against null model.

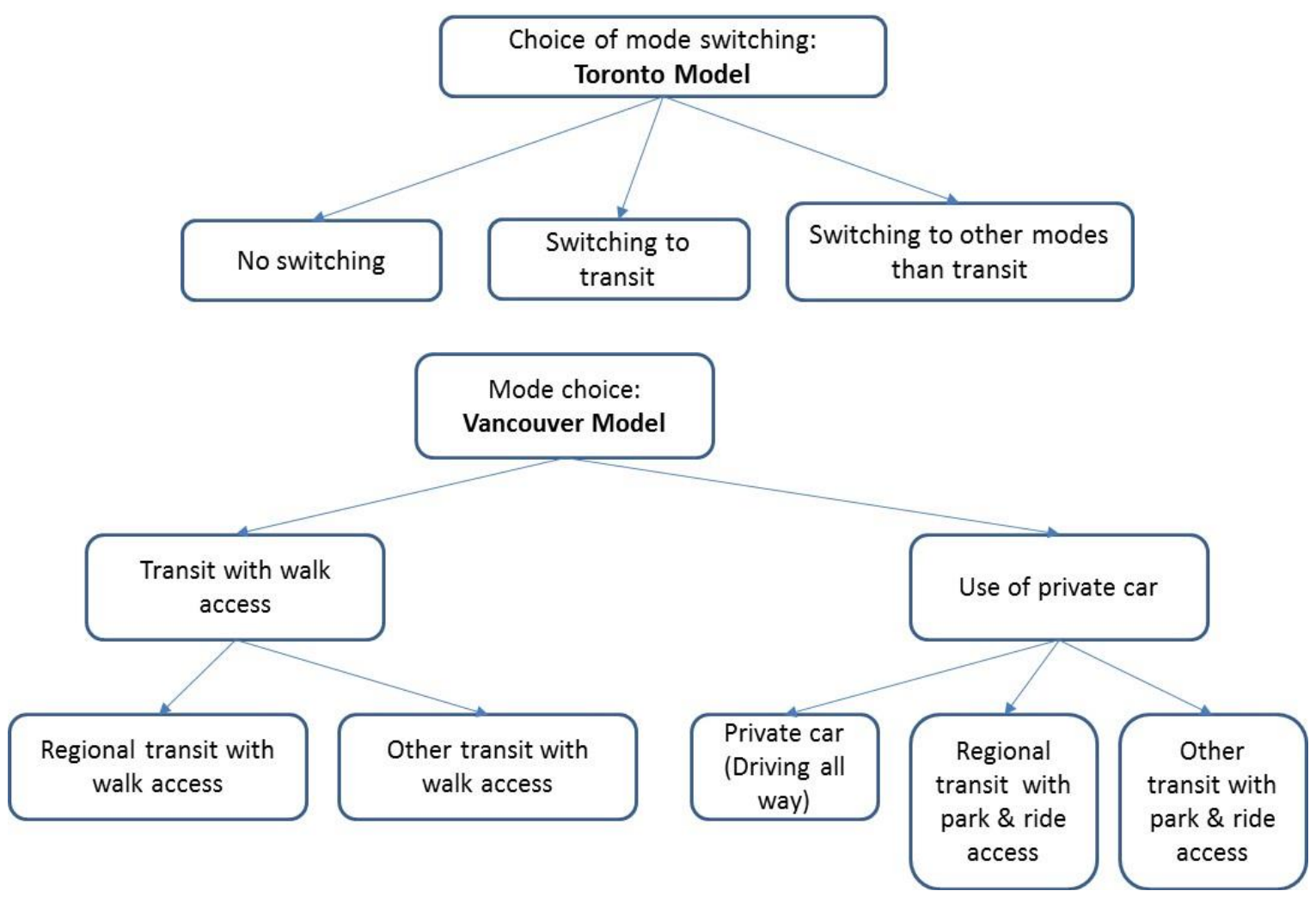

Figure 3: Choice alternatives of two models

The main objective of this paper is to investigate whether capturing such endogeneity would enhance the choice model parameter estimates or not. In other words, will there be any benefit of capturing such endogeneity by exploiting the joint model of choice and certainty ratings in enhancing choice model parameter estimates? I tend to answer these questions based on comparisons of choice model components of the joint models (of linear and quadratic entropy functions) with a univariate (only discrete) choice model. The comparison will be based on SP choice covariates and ratios of the key covariate parameters (marginal rate of substitutions).

Table 1 presents the estimated models using the Vancouver dataset, and Table 2 presents the estimated models by using the Toronto dataset. In both cases, three models are presented side by side. The first model considers a linear function of choice model entropy to explain the ordered SP certainty rating. The second model presents only the SP choice model without any certainty rating. The last one is a univariate discrete choice model.

In the case of the Vancouver model, correlations exist between the unobserved factors influencing the choices in SP choice tasks and corresponding certainty ratings but, the magnitudes of such correlations vary across the alternative SP choices. Apparently, the 
correlations between the unobserved factors influencing the choice of a private car option in an SP task and its corresponding SP rating is found to be the lowest in magnitude and has lower than $95 \%$ confidence limit. A possible explanation is that the context that leads to the choice of a private car is clearer than those of any other mode choices, and hence there are no apparent correlated residuals between the choice model utility and certainty rating functions. Among others, transit with walk access mode shows a higher correlation than transit with park \& ride options. In order to explain the impact of SP choice entropy on certainty rating, marginal effects of entropy are calculated from the ordered probit model.

In terms of entropy function, it seems that not all quadratic components of choice entropy have higher than 95 percent confidence limit in the ordered probability model of certainty ratings. However, comparing the likelihood values of the joint model, it is clear that the quadratic function improves the likelihood the function of the joint model. In order to better understand the effects of SP choice entropy on certainty rating, the marginal effects ${ }^{2}$ of choice entropy in the ordered certainty rating model is plotted in Figure 4. It explains that increasing choice entropy (resulting uncertainty from increasing choice task complexity) reduces certainty in SP choices. Highest choice entropy occurs in cases when all SP choice alternatives are perceived to be equally attractive to the respondent. In such cases, this empirical model reveals that the respondent is most likely to be unconfident in their SP choices.

The mean log likelihood values of choice model components of the joint model of Vancouver survey are higher than the corresponding single discrete choice model. This is reflected by higher Rho-squared values of the joint models. However, here is no apparent gain in Rho-squared values in the joint models of Toronto survey. Interestingly, Rho-squared values of the models estimated by using Toronto survey data are higher than those estimated by using Vancouver survey data. Explanation of such differences is, perhaps, in the choice contexts. In case of Toronto survey, the maximum number of choice alternatives is 3, but in case of Vancouver survey it is 5. It seems that goodness-of-fit gain happens for the choice context with high number of choice alternatives. However, in any case, there are model estimation efficiency is clear as higher number of estimated parameters are statistically significant. This is evident in Table 1 and 2 that the single choice model results in higher number of parameter estimates (of key variables, e.g. uncertainty in parking availability at the park $\&$ ride stations and frequency of schedule delay) having lower than $95 \%$ percent confidence limit.

\footnotetext{
${ }^{2}$ An approximate marginal effect calculation considering equivalent univariate confidence rating model is used. For example, for the confidence rating of ' $r$ ' and a linear entropy function $C_{k j}=\theta H_{s}$ the marginal effect of choice entropy $H_{s}$ on corresponding confidence rating of $r$ is

$M E_{r}=\phi\left(\mu_{r}-\theta H_{s}\right) \theta-\phi\left(\mu_{r-1}-\theta H_{s}\right) \theta$
}

Similarly, for a quadratic entropy function $C_{k j}=\theta H_{s}+\theta^{\prime} H_{s}^{2}$ the marginal effect of choice entropy $H_{s}$ on corresponding confidence rating of $r$ is

$M E_{r}=\phi\left(\mu_{r}-\theta H_{s}\right)\left(\theta+2 \theta^{\prime} H_{s}\right)-\phi\left(\mu_{r-1}-\theta H_{s}\right)\left(\theta+2 \theta^{\prime} H_{s}\right)$

Here, $\phi($.$) is the pdf of univariate normal distribution.$ 


\section{Table 1: Empirical Model of Mode Choice (Vancouver Dataset)}

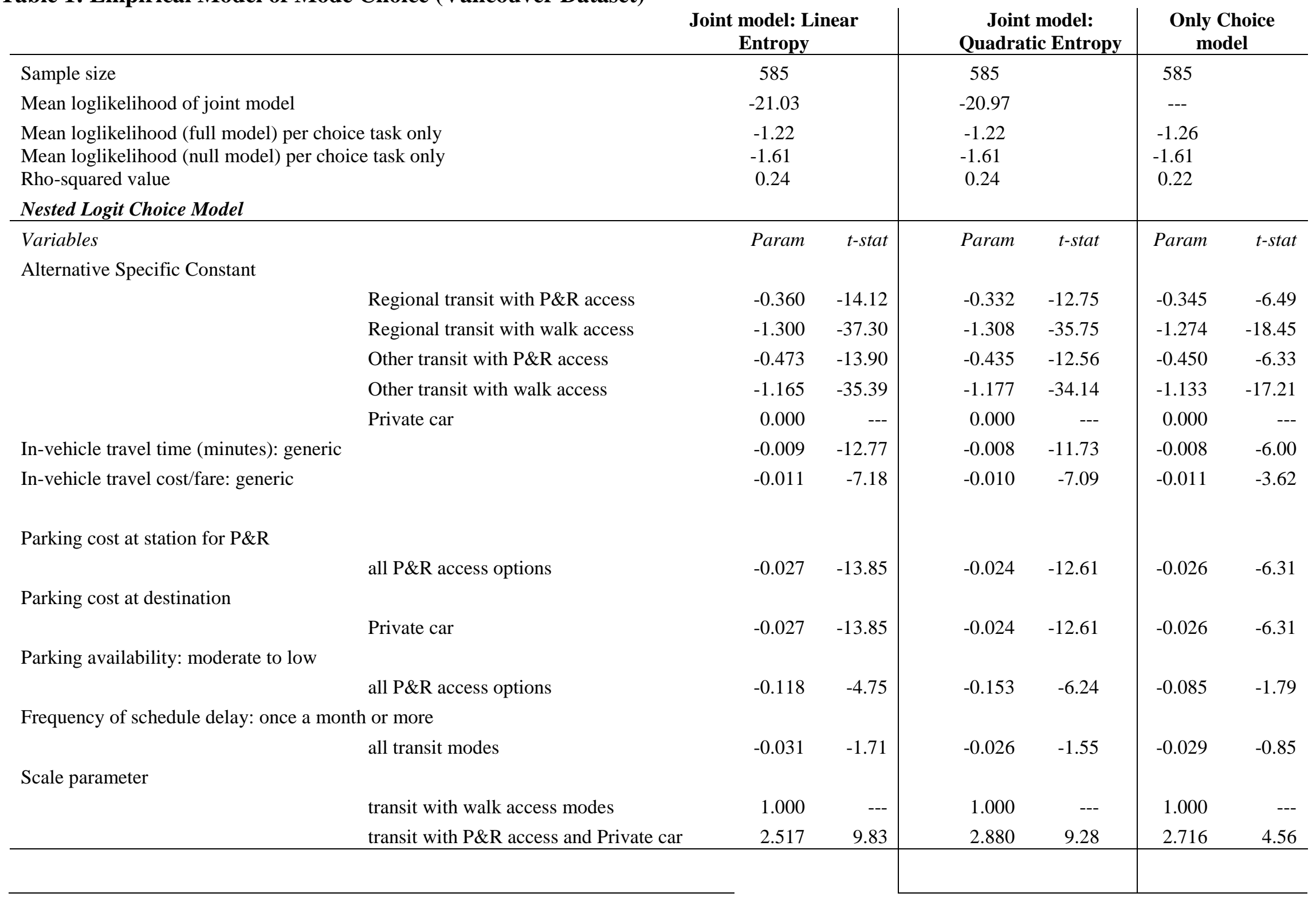




\section{Ordered Probit Certainty Rating Model}

Variables
Thresholds
Choice model entropy

Rating 1 (unsure)

Rating 2

Rating 3

Rating 4 (certain)

Regional transit with P\&R access

Regional transit with walk access

Other transit with $\mathrm{P} \& \mathrm{R}$ access

Other transit with walk access

Private car

Squared of choice model entropy

Regional transit with P\&R access

Regional transit with walk access

Other transit with P\&R access

Other transit with walk access

Private car

\section{Correlation coefficients}

Variables

Variables
Param

0.561

1.286

1.840

0.000

$-0.230$

$-0.445$

$-0.118$

$-0.341$

$-0.037$

\begin{tabular}{r|rr|r} 
& & & \\
\cline { 2 - 4 }$t$-stat & Param & $t$-stat & \\
10.37 & 3.533 & 7.94 & \\
25.60 & 4.227 & 9.47 & \\
37.24 & 4.773 & 10.68 & \\
--- & 0.000 & --- & \\
& & & \\
-4.12 & 5.193 & 6.77 & \\
-10.31 & 4.057 & 5.32 &
\end{tabular}

Regional transit with P\&R access

Regional transit with walk access

Other transit with P\&R access

Other transit with walk access

Private car

Ratio: In-vehicle Time by In-vehicle cost

$\begin{array}{lll}-1.71 & 1.836 & 1.67\end{array}$

$\begin{array}{lll}-9.31 & 1.849 & 2.20\end{array}$

$-0.41$

5.225

6.72

2.348

$-7.51$

$-1.699-5.53$

$-0.065 \quad-0.10$

$-0.179 \quad-0.41$

$-2.220 \quad-6.86$

Ratio: Parking cost by In-vehicle cost

Ratio: Parking availability by In-vehicle cost

Ratio: Frequency of schedule delay by In-vehicle cost

$\$ 2.795$ 


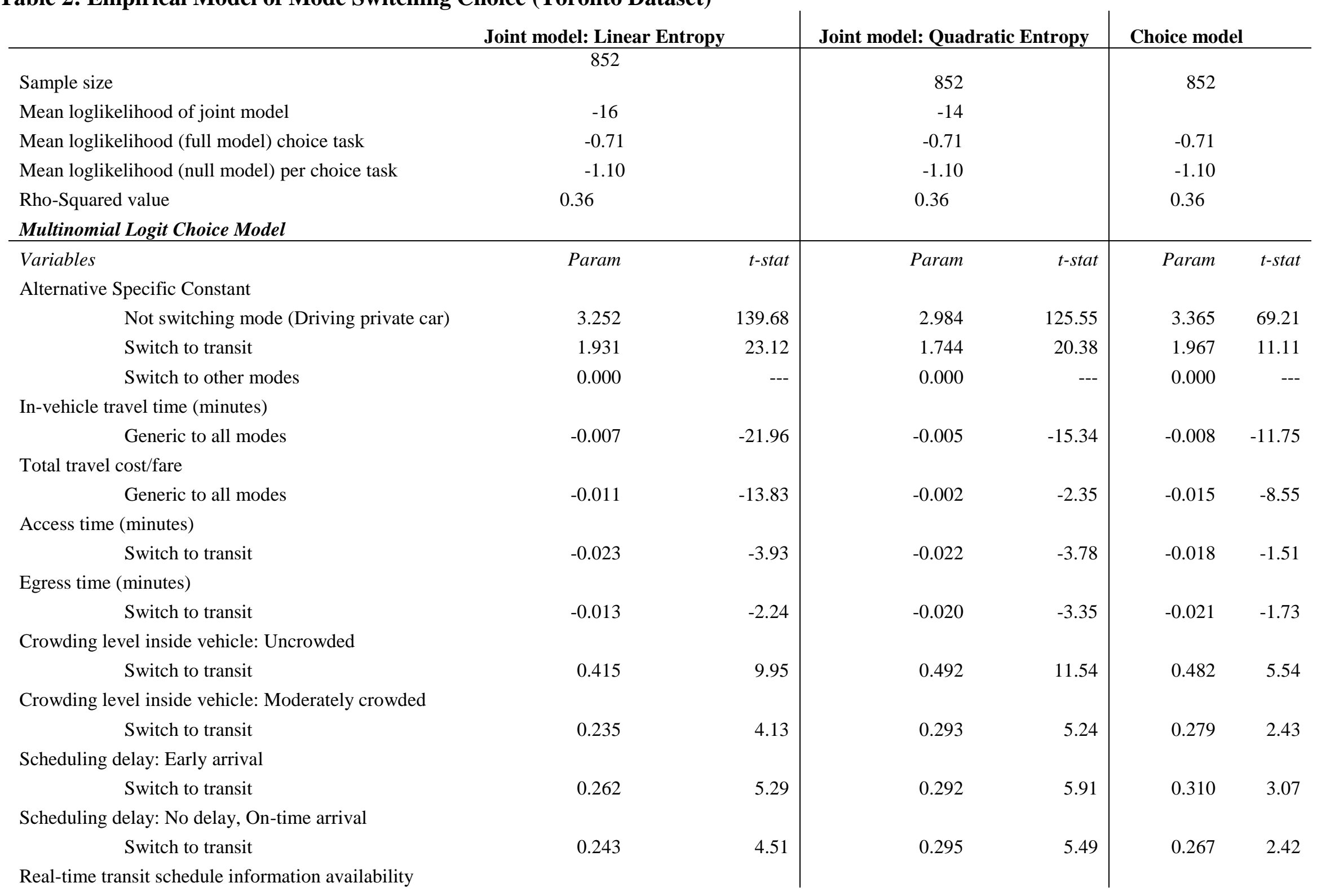


Switch to transit

Ordered Probit Certainty Rating Model

Variables

Thresholds

Very Weak or Moderately Weak

Neutral

Moderately strong

Very strong

Choice model entropy

Switch to transit

Switch to other modes

Squared of choice model entropy

Switch to transit

Switch to other modes
Not switching mode (Driving private car)

Not switching mode (Driving private car)

0.091

Param

0.327

0.913

1.593

0.000

0.913

0.097

$-0.700$

\section{Correlation coefficients}

\section{Variables}

Not switching mode (Driving private car)

Switch to transit

Switch to other modes

Ratio: In-vehicle Time by Travel cost

Ratio: Access time by Travel cost

Ratio: Egress time by Travel cost

Ratio: Uncrowded by Travel cost

Ratio: Moderately crowded by In-vehicle cost per $\mathrm{km}$

Ratio: Early arrival by In-vehicle cost per $\mathrm{km}$

Ratio: On-time arrival by In-vehicle cost per $\mathrm{km}$

Ratio: Real-time information by In-vehicle cost per $\mathrm{km}$

$-\$ 8.34$
0.037

0.84

0.074

0.81

\footnotetext{
* t-statistics higher than 1.64 indicates $95 \%$ confidence limit for one-tailed test and higher than 1.96 indicates $95 \%$ confidence limit for two-tailed test
} 


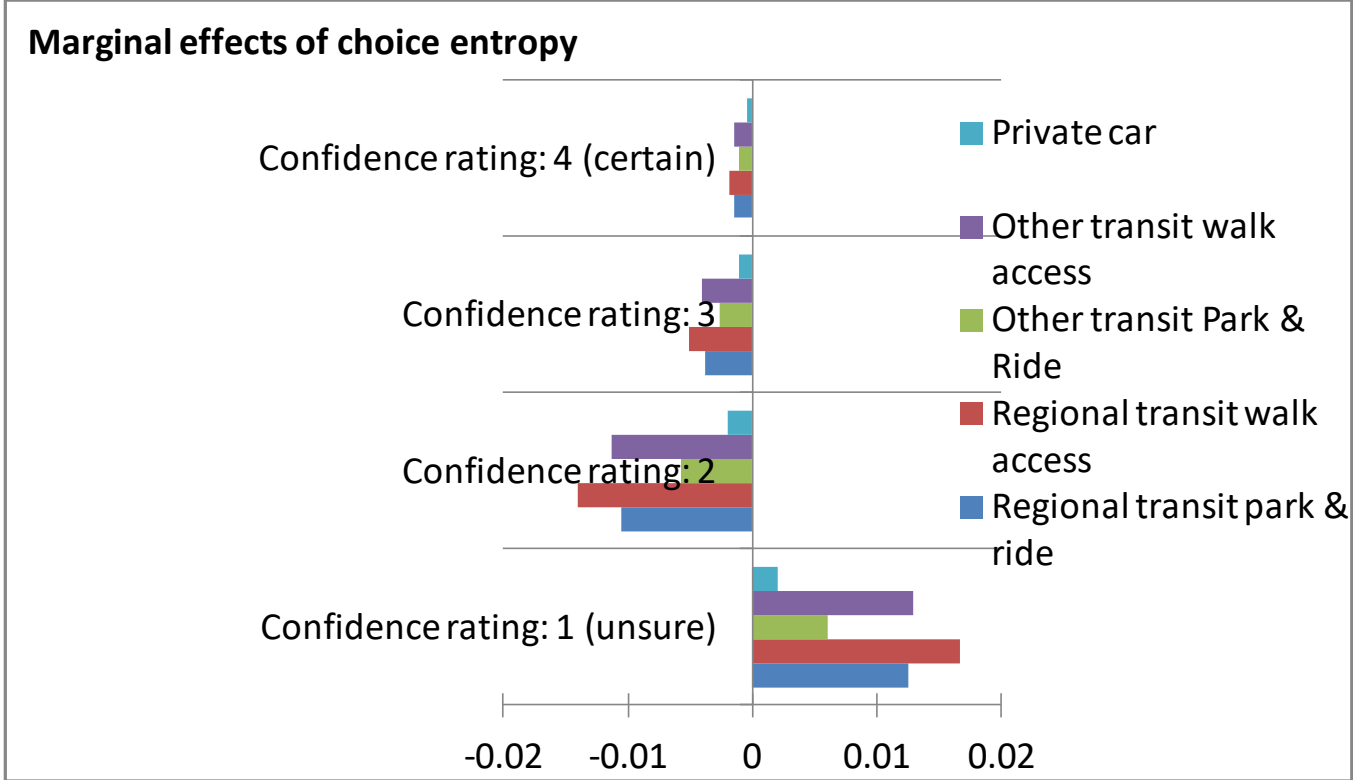

Figure 4: Marginal effects of choice entropy on certainty rating elicitation (Vancouver Data)

Confidence limits of parameter estimates improve (over 95\%) in the joint model, which clearly results in a gain in choice model estimation. Although the likelihood functions and statistical significances of choice model covariates improve in the joint models, most of the marginal rates of substitution between travel cost and other SP attributes remain almost the same except one. It seems that the univariate choice underestimates (with lower confidence limit) the willingness to pay for increasing parking availability in park \& ride stations than the joint models.

In the case of the Toronto model, non-zero correlations exist between the unobserved factors influencing the choices in SP choice tasks and corresponding certainty ratings. Similar to the Vancouver model, the lowest correlation is that between the unobserved factors influencing the choice of private car option (not switching to transit or other modes) in an SP task and its corresponding SP rating. Therefore, the same explanation prevails that the context leading to the choice of not switching from a private car is clearer than any other choice context; hence the magnitude of the correlation between the residuals drops and the certainty on estimated value drops too. Comparing the joint models, it seems that the quadratic entropy function model improves the likelihood of the joint model, perhaps by better fitting the certainty rating model (as all parameters of the certainty rating model component are non-zero in the quadratic function model). However, the likelihood values of the choice model components remain the same in all three models.

Comparing the joint model and the univariate choice model, it is clear that the univariate choice model results lower-confidence in parameter estimates of key variables e.g. access time, egress time and real-time information availability than the joint models. In terms of marginal rate of substitutions between travel cost and all other SP choice attributes, the univariate model clearly underestimates compared to those from the joint model. Among the joint models, the quadratic entropy function model gives the highest estimates all of these marginal rates of substitution. 


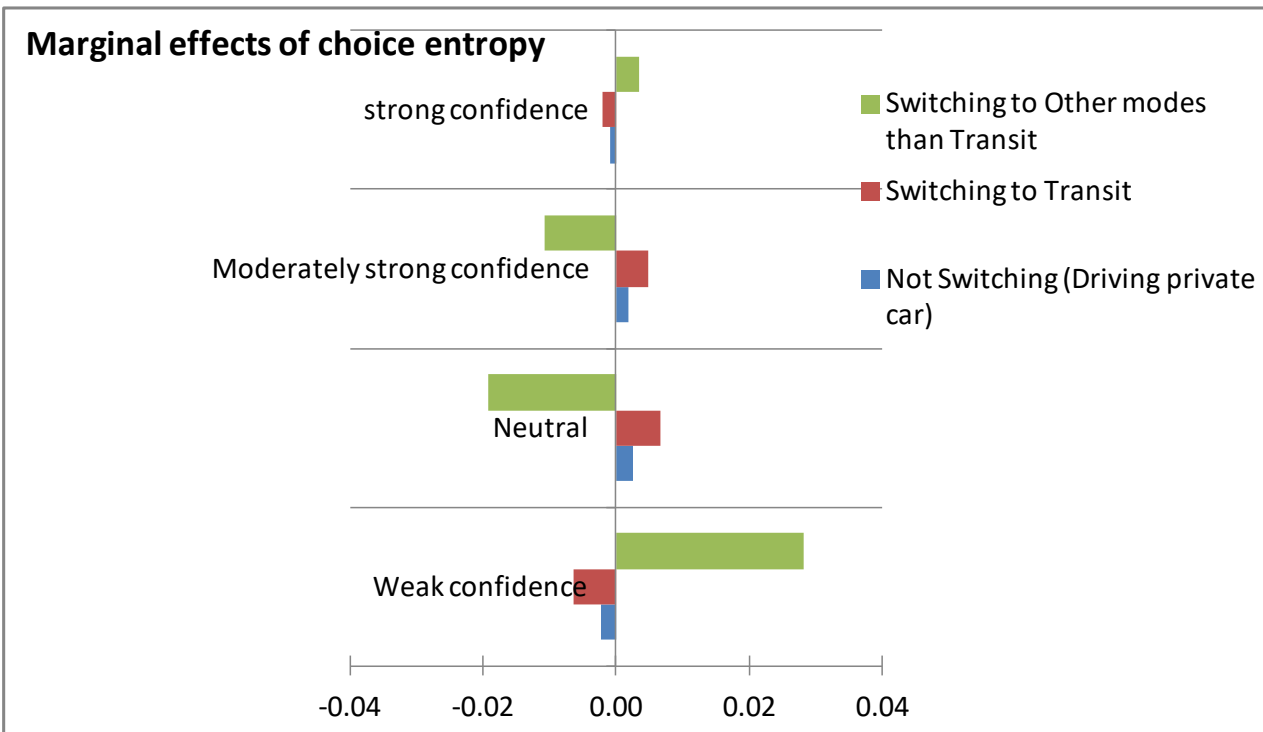

Figure 5: Marginal effects of choice entropy on certainty rating elicitation (Toronto Data)

In terms of the impact of choice entropy on SP certainty rating elicitation, Figure 5 presents the marginal effects of choice entropy calculated in a similar way as for the Vancouver model. However, unlike the Vancouver model, choice entropy affects different SP choice entropies differently. It seems that high choice entropy (higher complexity of SP choice tasks or equal attraction of all three SP choice options) makes the choice of switching to other modes either unconfident or highly confident. However, the opposite is true for the other two options: not switching or switching to transit. The actual context of the SP experiment is to blame in this case. In this case, switching to transit or not switching to transit options are clearly dominating over switching to other modes, as they are mostly non-motorized modes, which are in most cases not a very feasible alternative to many respondents. Between switching to transit and not switching option, choice entropy influences the certainty rating of switching to transit option. However, in both cases, higher entropy leads to a neutral or somewhat confident state than unconfident or highly confident states.

\section{Key Findings, Conclusions, and Recommendations for Future Studies}

The paper investigates the relationship between SP choice and corresponding elicited certainty rating. It employs two datasets collected in two cities. In one of the datasets (collected in Vancouver), a full mode choice model is tested and in the other (collected in Toronto) mode switching options are tested. In both cases, each of the six SP scenarios follows a four-point certainty rating elicitation ranging from unconfident to highly confident in making the SP choices. Considering there could be endogeneity between SP choices and corresponding elicitation of certainty rating, the paper proposes a joint econometric modelling structure. The proposed model considers that the SP certainty rating elicitation is directly influenced by SP choice uncertainty.

SP choice uncertainty, which may result from SP task complexities (reflected through combinations of SP alternative choice attribute levels), is specified by SP choice entropy. It is 
assumed that either linear or quadratic functions of SP choice entropy work as covariate functions of the ordered certainty ratings. In addition, the proposed model considers unrestricted correlations between unobserved factors influencing an SP choice and corresponding certainty rating. Empirical models are estimated for both datasets and are evaluated to investigate whether the joint estimation of the SP certainty rating model with the SP choice model has any influence on the choice model.

Empirical models confirm that significant endogeneity exists between SP choices and corresponding certainty rating elicitations. However, the most important and common gain in the joint estimation is the improvement in the choice model parameter significances. In both empirical models, it is clear that exploiting the endogeneity between SP choices and corresponding certainty rating elicitation improves the choice model estimation efficiency. In both empirical investigations, it is found that the confidence limits of parameter estimate of multiple SP choice attribute increase in the joint choice and certainty rating model than those in the univariate models. Also, depending on the SP experiment type, it is also evident that overall goodness-of-fit of the choice model component in the joint model may also increase (compared to that of the univariate choice model) because of the joint econometric model structure.

Comparing the choice model components of the joint model and the only SP choice model, it is evident that an increase in choice model estimation efficiency may also result in changes in parameter estimates (i.e. marginal effects). In both empirical cases, it is found that the univariate SP choice model underestimates the marginal rate of substitutions of SP choice attributes. However, the magnitudes of the underestimations vary by SP choice tasks as well as specific choice attributes. These findings empirically justify Manski's (1995) assertion that incorporation of the respondent's elicited certainty into the choice model estimation process would improve model estimation accuracy. In addition, it is also evident that the measure of choice entropy of SP choice tasks effectively explains the corresponding certainty rating elicitation. In this paper, both linear and quadratic functional forms of entropy functions are tested. It is found that the quadratic functional form of entropy offers a better fit for the certainty rating elicitation choices.

Overall, the proposed methodology provide a better choice model estimation procedure by jointly estimating SP choice and SP confidence rating. Since, SP confidence ratings are often collected along with SP choice data, such joint estimation exploits the elicited confidence rating information to generate better parameter estimates. In many practical project, such additional information (elicited confidence ratings) are just overlooked or choice datasets are arbitrarily trimmed based on assumed threshold values of elicited confidence ratings. The advantage of the proposed methodology is that it generates a better choice model than can be used for choice forecasting and/or sensitivity analyses like any other choice model. In any case, forecasting of confidence rating is not necessary for the application of the estimated choice model as nothing of the elicited confidence rating enter into the choice mode as inputs.

Research presented in this paper can be extended on several fronts. Effects of jointly estimating revealed and stated preference models along with certainty ratings are clearly important avenues for further research. Whether integrating RP choice model with SP choice and certainty rating would affect choice model parameter estimations is worth investigating. Similarly, effects of further accommodation of preference heterogeneity and heteroskedasticity in the context of joint 
RP-SP and certainty rating model requires the attention of choice model researchers. So, these are considered recommendations for further research. Also, the possible moderating role of elicited confidence rating between presented SP choice set and SP choices needs further investigation. Since the main purpose of this paper is to improve the choice model, only choice entropy (task complexity) is used to explain the certainty ratings. However, one can also expect that in addition to task complexity, there could be other factors, e.g., socio-economic and personal attributes that influence certainty rating. Thus, such effort is recommended for future research. Also, in this current approach, the main objective was to explain elicited choice uncertainty through SP choice entropy measures. However, to justify findings of Caussade et al. (2005) that SP task complexities affect SP choice variances; one can specify the SP choice scale parameter though the same entropy functions as used by Swait and Adamowicz (2001a). This would further complicate the model estimation process (as reported by Dekker et al. 2016), but it would be worth investigating as a next step.

In terms of collecting information of certainties, the paper used two survey datasets that used the slightly different approach of collecting SP certainty information. The Toronto dataset used qualitative options and the Vancouver dataset used a numerical scale. However, the paper does not investigate different ways of SP certainty elicitation process. However, based on empirical investigations by using two datasets, it is understandable that a numerical scale is less confusing than qualitative options of elicitation. For example, in the Toronto dataset, the option of 'neutral' may be interpreted by a respondent as neutral between two choice alternatives or so. Even in the case of the numerical scale of elicitation, the Vancouver dataset used a 4-point scale. I believe that further research is necessary to investigate different approaches to certainty elicitation process.

After all, the proposed methodology of exploiting elicited confidence ratings of SO survey used standard multinomial logit (MNL) and nested logit (NL) model for the joint econometric formulations. However, one could also presume that the benefit gain can be different for more advanced choice models, e.g. latent class discrete choice model and or generalized mixed logit models. As a logical first step, I used standard MNL and NL model, but it will be a straight forward extension of estimating more advanced choice model for the joint formulation. I believe, comparative study on the effects of choice model formulation (standard model versus more advanced model) on benefit gain in the joint discrete choice and confidence rating model is worth investigating. So, this is considered as a next step of current investigation.

\section{Acknowledgements}

This research was funded by an NSERC Discovery Grant. The author acknowledges the contributions of Dr. Ahmed Idris and Dr. Amer Shalaby from the University of Toronto in the data collection project of the Toronto survey; Jesse Coleman and Blair Smith from IBI for facilitating access to the Vancouver dataset and TransLink for allowing the use of Vancouver datasets for this research. An earlier version of the paper was presented at the $95^{\text {th }}$ Annual Meeting of Transportation Research Board (TRB), Washington DC. The paper is benefited by the comments from the audiences in the TRB session and three anonymous reviewers of TRB. Finally, the author acknowledges the reviews and comments of two anonymous reviewers of the 
Journal of Choice Modelling in improving the quality of the paper. However, the views and explanations expressed in this paper are only of the author himself.

\section{References}

1. Aptech System. 2015. The GAUSS ${ }^{\mathrm{TM}}$ Mathematical and Statistical Systems. http://www.aptech.com/products/gauss-mathematical-and-statistical-system/

2. Arentze, T., Borgers, A., Timmermans, H., DelMistro, R. 2003. Transport stated choice responses: Effects of task complexity, presentation format and literacy. Transportation Research Part E 39: 229-244

3. Beck, M.J., Rose, J.M., Hensher, D.A. 2013. Consistently inconsistent: The role of certainty, acceptability and scale in automobile choice. Transportation Research Part E 56:81-93

4. Bemmaor, A. 1995. Predicting behaviour from intention-to-buy measures: the parametric case. Journal of Marketing Research 32: 176-191

5. Brouwere, R., Dekker, T., Rolfe, J., Wildle, J., 2010. Choice certainty and consistency in repeated choice experiment. Environmental Resource Economics 46: 93-109

6. Carson, R. Louviere, J. 2011. A common nomenclature for stated preference elicitation approach. Environ Resource Economics 49: 539-559

7. Caussade, S., Ortuzar, J.d.D, Lizzi, L.I., Hensher, D.A. 2005. Assessing the influence of design dimensions on stated choice experiment estimates. Transportation Research Part B 39: 621-640

8. Champ, P. A., Bishop, R. C. 2001. Donation payment mechanisms and contingent valuation: An empirical study of hypothetical bias. Environmental and Resource Economics 19(4): 383-402

9. Champ, P. A., Bishop, R. C., Brown, T. C., McCollum, D. W. 1997. Using donation mechanisms to value non-use benefits from public goods. Journal of Environmental Economics and Management 33(2): 151-162

10. Dekker, T., Hess, S., Brouwe, R., Hofkes, M. 2016. Decision uncertainty in multiattribute stated preference studies. Resource and Energy Economics 43: 57-73

11. Ethier, R.G., Poe, G. L. Schulze, W. D., Clark J. 2000. A comparison of hypothetical phone and mail contingent valuation responses for green-pricing electricity programs. Land Economics 76(1): 54-67

12. Habib, K.M.N., Swait, J., Salem, S. 2014. Using repeated cross sectional travel surveys to increase forecasting robustness: Accounting for changing mode preferences. Transportation Research Part A 67: 110-126

13. Hanemann, W.M., Kristrom, B., Li, C. 1995. Nonmarket valuation under preference uncertainty: Econometric models and estimation. The paper presented at the Annual Meeting of the European Association of Environmental and Resource Economics, Umea, Sweden, June 17-20, 1995

14. Hensher, D. Ho, C. 2014. Identifying a behaviourally relevant choice set from stated choice data. Transportation (Forthcoming). DOI 10.1007/s11116-014-9572-z

15. Hensher, D., Rose, J.M., Beck, M.J. 2012. Are there specific design elements of choice experiments and types of people that influence choice response certainty? Journal of Choice Modelling 5(1): 77-97 
16. Idris, A.O., Habib, K.M.N., Shalaby, A. 2014. Dissection the role of transit service attributes in attracting commuters: Lessons from a comprehensive RP-SP study on commuting mode switching behaviour in Toronto. Transportation Research Record 2415: 107-117

17. Lee, L. F. 1983. Generalized econometric models with selectivity. Econometrica 51 (2): 507-512

18. Li, C-H., Mattsson, L. 1995. Discrete choice under preference uncertainty: An improved structural model for contingent valuation. Journal of Environmental Economics and Management 28: 256-269

19. Louviere, J., Flynn, T.N., Carson, R.T. 2010. Discrete choice experiments are not conjoint analysis. Journal of Choice Modelling 3(3): 57-72

20. Lundhede, T.H., Olsen, S.B., Jacobson, J.B., Thorsen, B, J. 2009. Handling respondent uncertainty in choice experiments: Evaluating recording approaches against explicit modelling of uncertainty. Journal of Choice Modelling 2(2): 118-147

21. Manski, C. 1995. Identification problems in the social sciences. Harvard University Press, Cambridge, MA, USA

22. Manski, C. 1999. Analysis of choice experiments in incomplete scenarios. Journal of Risk Uncertainty. 19:49-66

23. Noreheim B. 2001. Stated preference surveys. 2001. Do we have confident tests for the results? Paper presented at the International Conference on Transport Survey Quality and Innovation. South Africa, Aug 5-10, 2001

24. Olsen, S.B., Lundhede, T.H., Jacobson, J.B., Thorsen, B.J. 2011. Tough and easy choices: Testing the influence of utility differences on stated certainty-in-choice in choice experiments. Environmental Resource Economics 49: 491-510

25. Ready, R., Whitehead, J., Blomquist, G., 1995. Contingent valuation when respondents are ambivalent. Journal of Environmental Economics and Management 29 (2), 181-197

26. Rose, J., Bliemer, M. 2009. Constructing efficient stated choice experimental designs. Transport Reviews 29(5): 587-617

27. Rose, J., Bliemer, M. 2013. Sample size requirements for stated choice experiments. Transportation 40: 1021-1041

28. Rose, J., Beck, M.J., Hensher, D.A. 2015. The joint estimation of respondent-reported certainty and acceptability with choice. Transportation Research Part A 71: 141-152

29. Shaikh, S.L, Sun, L., Kooten, G.C.v. 2007. Treating respondent uncertainty in contingent valuation: A comparison of empirical treatments. Ecological Economics 62: 115-125

30. Shannon, C.E., 1948. A mathematical theory of communication. Bell System Technology Journal 27: 379-423

31. Swait, J., Adamowicz, W. 2001a. Choice environment, market complexity, and consumer behaviour: A theoretical and empirical approach for incorporating decision complexity into models of consumer choice. Organizational Behaviour and Human Decision Processes 86 (2): 141-167

32. Swait, J., Adamowicz, W. 2001b. The influence of task complexity on consumer choice: A latent class model of decision strategy switching. Journal of Consumer Research 28: 135-148 